\title{
SUPPLEMENT to The effect of secondary ice production parameterization on the simulation of a cold frontal rainband
}

Sylvia C. Sullivan ${ }^{1,2}$, Christian Barthlott ${ }^{1}$, Jonathan Crosier $^{3}$, Athanasios Nenes ${ }^{2,4,5}$, and Corinna Hoose ${ }^{1}$

${ }^{1}$ Institute of Meteorology and Climate Research, Karlsruhe Institute of Technology, Karlsruhe, Germany

${ }^{2}$ Department of Chemical and Biomolecular Engineering, Georgia Institute of Technology, Atlanta, GA 30332, USA

${ }^{3}$ School of Earth, Atmospheric, and Environmental Studies, University of Manchester, Manchester, UK

${ }^{4}$ ICE-HT, Foundation for Research and Technology, Hellas, 26504 Patras, Greece

${ }^{5}$ Institute of Environmental Research and Sustainable Development, National Observatory of Athens, 15236, Palea Penteli, Greece

Correspondence to: S. Sullivan (scs2229@ columbia.edu), A. Nenes (athanasios.nenes@gatech.edu), C. Hoose (corinna.hoose@kit.edu) 


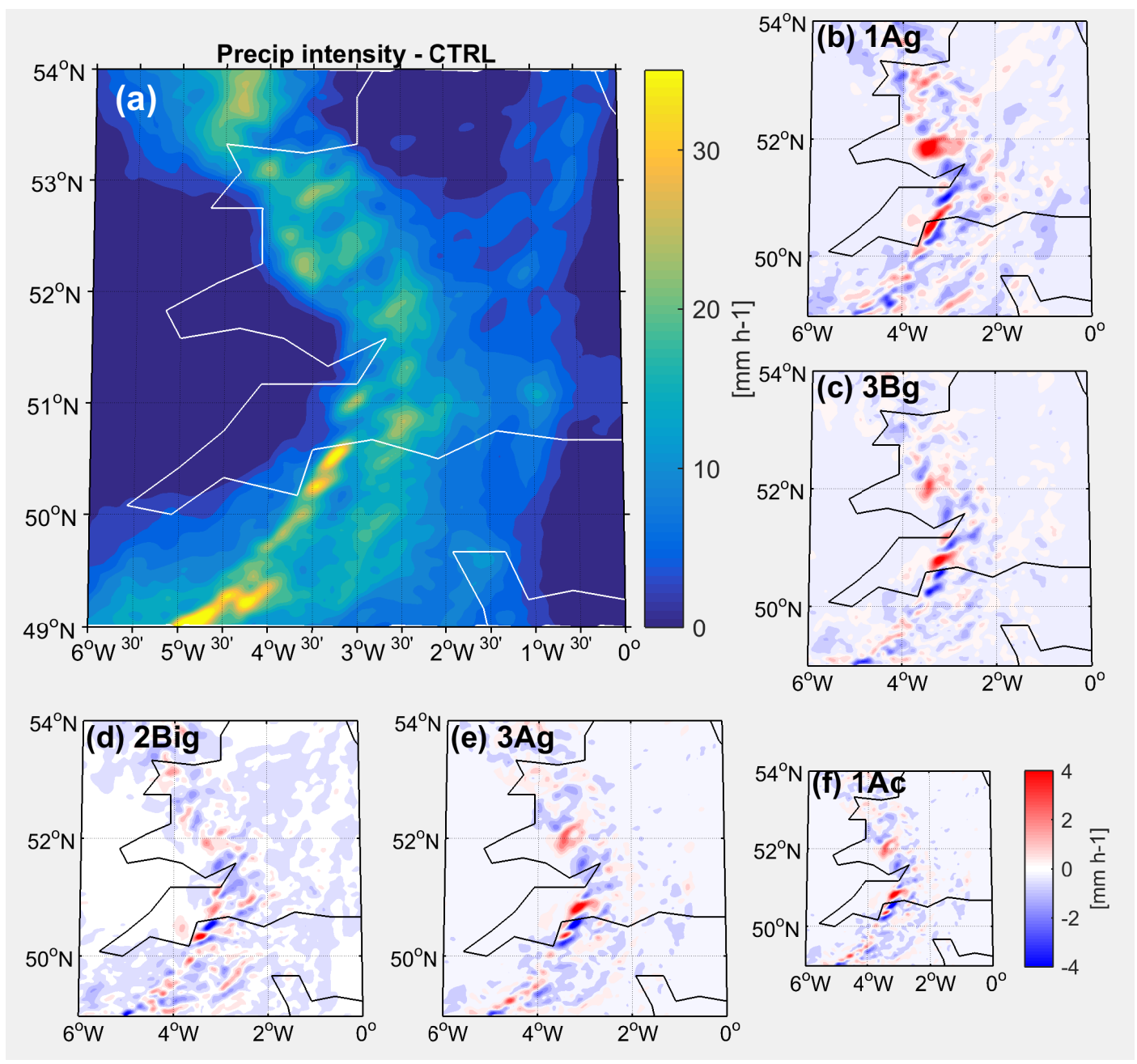

Figure S1. Panel a shows the spatial distribution of precipitation rate in the control simulation for a subdomain centered at CFARR between 1800 and 1830 UTC, as the rainband begins to pass over the UK. Panels $b$ though $f$ show the deviations of precipitation rate in five of the simulations with the secondary ice parameterizations in place. 

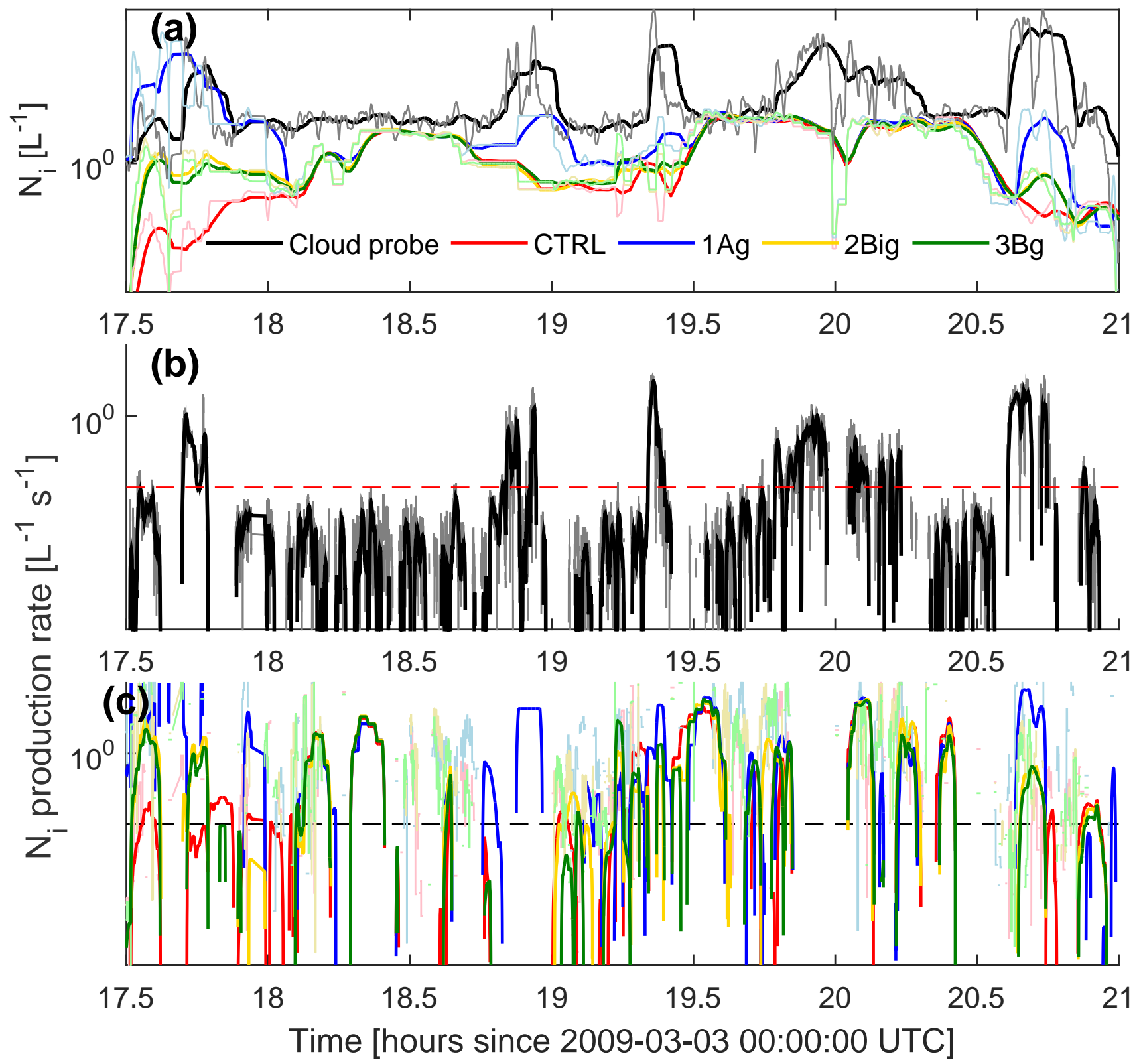

Figure S2. Additionally-smoothed ICNC time series are shown in panel a from CIP-15 observations (gray - 30-point running mean; black 300-point running mean) and the CTRL, 1Ag, 3Bg, and 2Big simulations (pale lines - 30-point running mean; bold lines - 300-point running mean). Estimations of ice production rate are shown in panel b from observations and in panel $\mathrm{c}$ from simulations, with the running means calculated and visualized as in panel a. The dashed lines in panels $\mathrm{b}$ and $\mathrm{c}$ indicate the $0.1 \mathrm{~L}^{-1} \mathrm{~s}^{-1}$ level around which many literature values fall. 


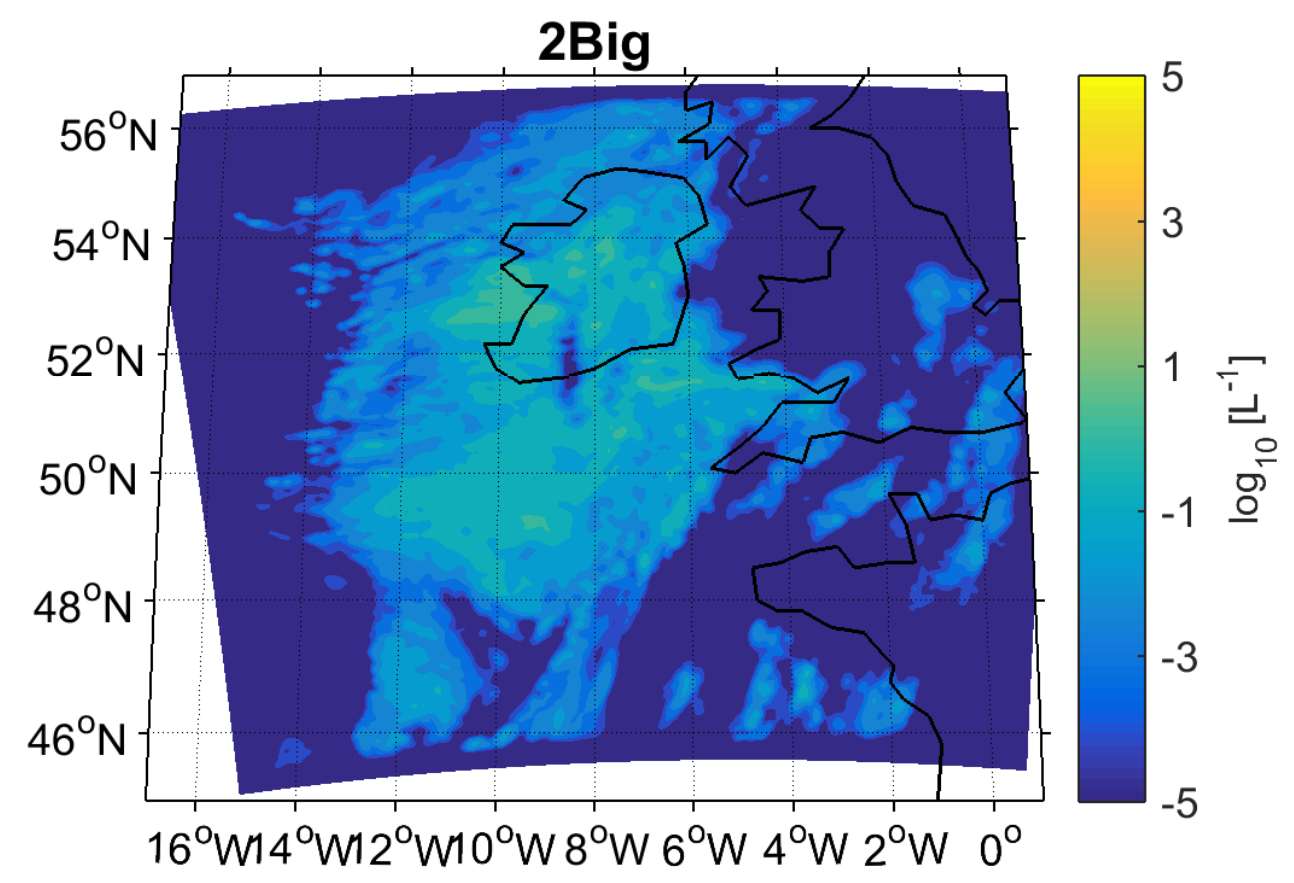

Figure S3. Map of secondarily-produced ice from the $2 \mathrm{Big}$ simulation at the pressure level where $\bar{T} \approx 258 \mathrm{~K}$, as the rainband began to pass over the UK. $N_{i, s e c}$ is a cumulative value between 1800 and 1900 UTC and the colorbar is logarithmic. 